“@ 2018 IEEE. Personal use of this material is permitted. Permission from IEEE must be obtained for all other uses, in any current or future media, including reprinting/republishing this material for advertising or promotional purposes, creating new collective works, for resale or redistribution to servers or lists, or reuse of any copyrighted component of this work in other works." 


\title{
A Wideband Base Station Antenna with Stable Radiation Pattern
}

\author{
Haihan Sun, Can Ding, Tianyu Yang, Y. Jay Guo, and Peiyuan Qin \\ Global Big Data Technologies Centre, University of Technology Sydney, Sydney, Australia \\ Haihan.Sun@student.uts.edu.au
}

\begin{abstract}
This paper presents the configuration and experimental results of a novel wideband dual-polarized base station antenna with superior performance. The proposed antenna consists of four electric folded dipoles arranged in an octagon shape that are excited simultaneously for each polarization. Experimental results show that this element has a wide bandwidth of $46.4 \%$ from $1.69 \mathrm{GHz}$ to $2.71 \mathrm{GHz}$ with $\geq 15$ dB return loss. Across this wide band, the variations of the halfpower-beamwidths (HPBWs) of the two polarizations are all within $66.5^{\circ} \pm 5.5^{\circ}$, the port-to-port isolation is $>28 \mathrm{~dB}$, and the cross-polarization discrimination (XPD) is $>25 \mathrm{~dB}$.
\end{abstract}

Keywords- base station antenna, dual-polarization, stable radiation patterns.

\section{INTRODUCTION}

The environmental demands and the rising costs of acquiring new sites for base stations along with the need for increased capacity of mobile networks have led to ever more stringent requirements on base station antennas. These include wide bandwidth, high port-to-port isolation, high crosspolarization discrimination, and beamwidth consistency. There are many challenges in meeting all these specifications simultaneously. As a result, there have been increasing research activities in base station antennas recently [1]-[5]. Among all the base station antenna elements employed in the mobile industry, cross-dipole is the most widely used antenna element. Broad impedance bandwidth with excellent matching can be achieved by utilizing strong mutual coupling between the two dipoles [1]. However, their radiation patterns intrinsically vary significantly across the operation band [2], as the patterns are mainly attributed to the radiation of one dipole for each polarization. In order to stabilize the radiation pattern, shaped ground and walls [3]-[5] were introduced. These techniques are suitable for single band antennas, but they can cause undesirable scattering in the other band in dual band antenna systems and therefore should be avoided.

In this paper, a dual-polarized antenna comprising four folded dipoles with superior performance that meets all the specifications of $4 \mathrm{G}$ base station antenna elements is proposed. It employs four folded dipoles that are fed by two unique feed networks to realize the two polarizations. Experimental results show that the proposed antenna is well matched across a wide band from $1.69 \mathrm{GHz}$ to $2.71 \mathrm{GHz}$ for both the two polarizations. Stable radiation patterns are also achieved without any modification of the ground plane.

\section{ANTENNA CONFIGURATION}

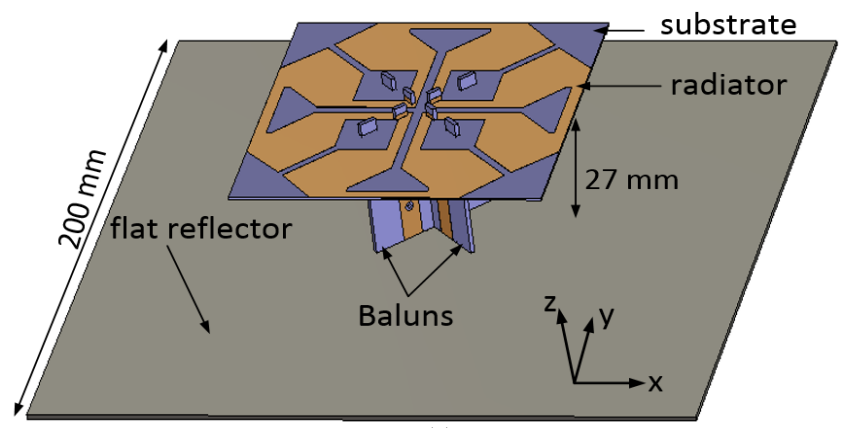

(a)

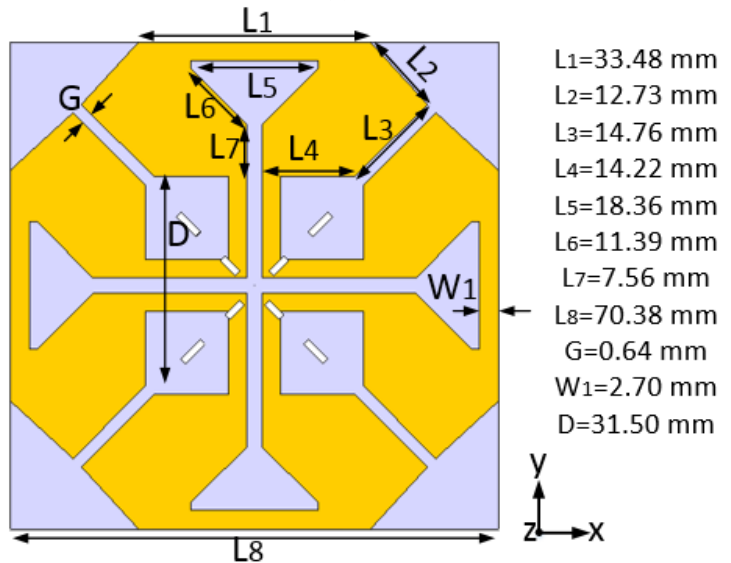

(b)

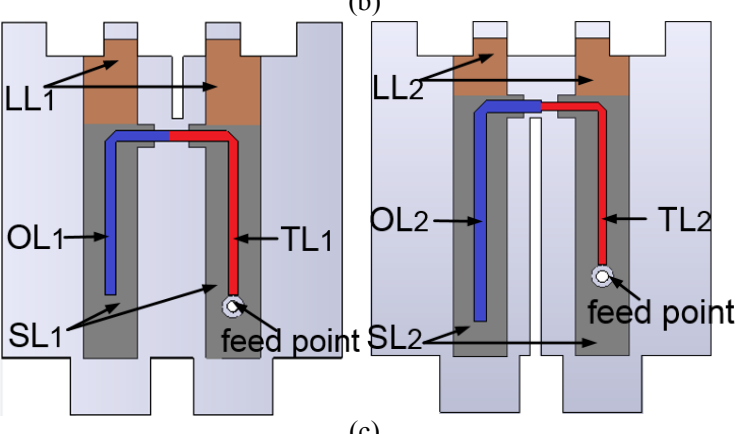

(c)

Fig. 1 (a) Perspective view of the proposed antenna, (b) top view of the aperture, and (c) configuration of the feed structure.

The configuration of the broadband dual-polarized antenna element is shown in Fig. 1. It consists of four folded dipole radiators, two microstrip feed structures, and a flat square metallic plate as reflector. The four folded dipoles are printed 


\section{$3^{\text {rd }}$ Australian Microwave Symposium, Brisbane, Australia, 6 - 7 February 2018}

on the substrate and are closely arranged in the shape of an octagon. The folded dipole arms are fed by parallel lines to four points forming a square at the center of the board. They are excited by two feed structures in the diagonal directions to realize the $\pm 45^{\circ}$ polarizations. As shown in Fig. 1(c), each feed structure consists of a link line (LL), a short line (SL), a transmission line (TL), and an open line (OL). The TL and OL are printed on the front layer, whereas LL and SL are printed on the back layer of the substrate. When one polarization is excited, all the four folded dipoles are used and are excited in two pairs of series connected dipoles. The two feed structures are mounted between the radiator and the reflector. The distance between the radiator and the reflector is $27 \mathrm{~mm}(0.20 \lambda$ at $2.2 \mathrm{GHz}$ ). All the substrates employed in this work as shown in Fig. 1 are FR4 substrate with a dielectric constant of 4.3. The thicknesses of substrate for the radiator and feed structures are $0.5 \mathrm{~mm}$ and $1.0 \mathrm{~mm}$, respectively.

\section{Simulation AND MEASUREMENT RESUlts}

The prototype was also fabricated and tested. Fig. 2 plots the simulated and measured S-parameters of the two ports. The measured bandwidth for $\left|\mathrm{S}_{11}\right| /\left|\mathrm{S}_{22}\right| \leq-15 \mathrm{~dB}$ is $46.4 \%$ from 1.69 $\mathrm{GHz}$ to $2.71 \mathrm{GHz}$, which covers the required bandwidth for base station application. The measured port-to-port isolation is around $30 \mathrm{~dB}$ over the entire operation frequency band. The simulated and measured radiation patterns at 1.7 and $2.7 \mathrm{GHz}$ in the xoz-plane are shown in Fig. 3. Only the results of $+45^{\circ}$ polarization are shown here as the antenna is symmetrically arranged and the radiation patterns of the two polarizations are very similar. The simulated and measured patterns agree very well. The measured cross polarization level is $>25 \mathrm{~dB}$ at the main lobe in the horizontal plane. The simulated and measured front-to-back ratios are $>22 \mathrm{~dB}$ and $>20 \mathrm{~dB}$, respectively. Fig. 4 shows the simulated and measured half-power beamwidths (HPBWs) of the antenna for both the two polarizations. The simulated and measured HPBWs are $64.5^{\circ} \pm 5.0^{\circ}$ and $66.5^{\circ} \pm 5.5^{\circ}$ for the two polarization patterns, respectively. The measured results demonstrate that this antenna has very stable radiation patterns across a wide bandwidth. The simulated and measured gains are also shown in Fig. 4. The simulated gain varies from $9.3 \mathrm{dBi}$ to $10.1 \mathrm{dBi}$, and the measured gain varies from $9.0 \mathrm{dBi}$ to $10.7 \mathrm{dBi}$ within the targeted bandwidth.

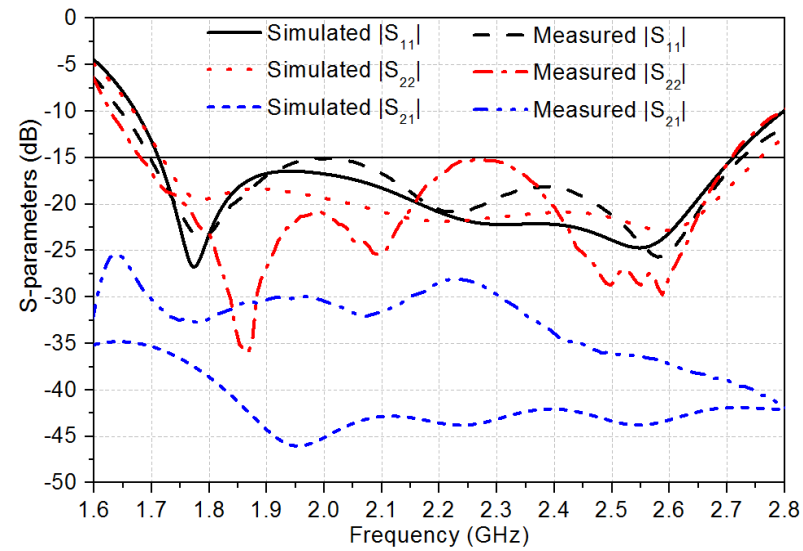

Fig. 2 Simulated and measured S-parameters.
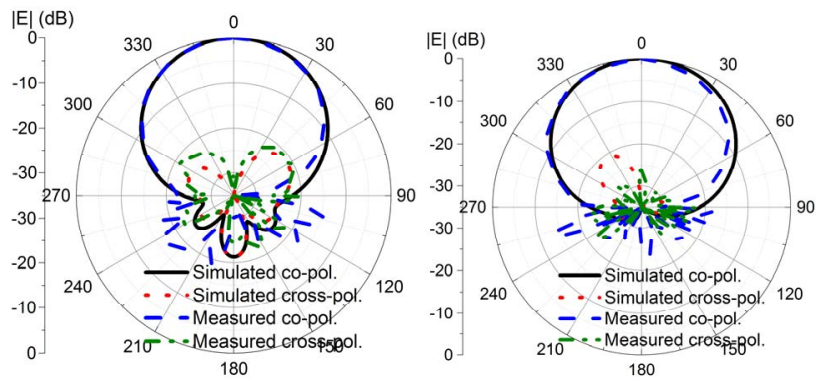

Fig. 3 Simulated and measured radiation patterns in the horizontal plane at (a) $1.7 \mathrm{GHz}$, and (b) $2.7 \mathrm{GHz}$.

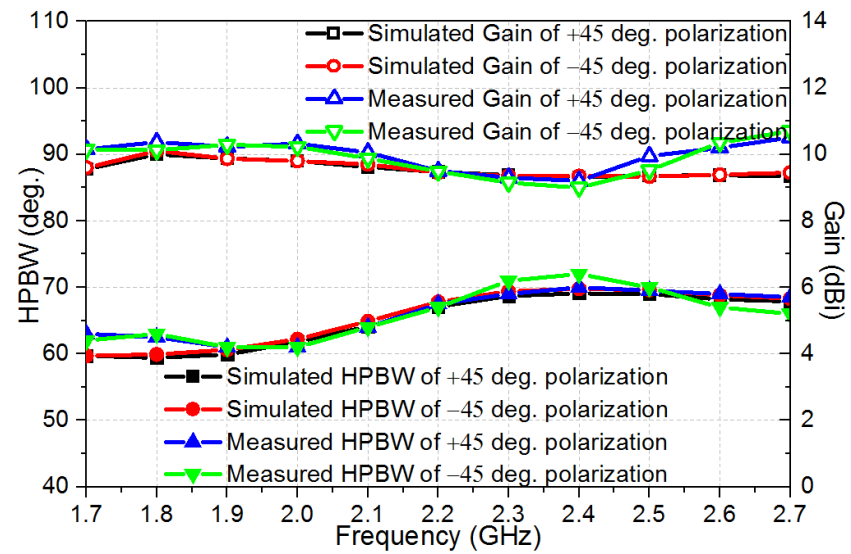

Fig. 4 Simulated and measured HPBWs and gains.

\section{CONCLUSION}

A wideband $\pm 45^{\circ}$ dual-polarized antenna element comprising four simultaneously excited folded dipoles is proposed for base station applications. The antenna features excellent radiation performance including wide bandwidth, high port-to-port isolation, low cross polarization, and stable radiation patterns. The element requires no walls or other external beam shaping and is ideal for use in dual band antenna systems.

\section{REFERENCES}

[1] Y. Liu, H. Yi, F. W. Wang and S. X. Gong, "A novel miniaturized broadband dual-polarized dipole antenna for base station," IEEE Antennas Wireless Propag. Lett., vol. 12, pp. 1335-1338, 2013.

[2] Y. Gou, S. Yang, J. Li, and Z. Nie, "A compact dual-polarized printed dipole antenna with high isolation for wideband base station applications," IEEE Trans. Antennas Propag., vol. 62, no. 8, pp. 43924395, Aug. 2014.

[3] Y. Cui, R. Li, and H. Fu, "A broadband dual-polarized planar antenna for 2G/3G/LTE base stations," IEEE Trans. Antennas Propag., vol. 62, no. 9, pp. 4836-4840, Sept. 2014.

[4] H. Huang, Y. Liu, and S. Gong, "A broadband dual-polarized base station antenna with sturdy construction," IEEE Antennas Wireless Propag. Lett., vol. 16, pp. 665-668, 2017.

[5] Q. X. Chu, D. L. Wen, and Y. Luo, "A broadband $\pm 45^{\circ}$ dual-polarized antenna with Y-shaped feeding lines," IEEE Trans. Antennas Propag., vol. 63 , no. 2 , pp. 483-490, Feb. 2015. 